\title{
离子液体捕集二氧化硫气体的研究进展
}

\author{
崔国凯，赵宁，张峰涛，王键吉*
}

河南师范大学化学化工学院, 河南省绿色化学重点实验室, 精细化学品绿色制造河南省协同创新中心, 绿色化学介质与反应教育部重点实 验室, 新乡 453007

* 联系人, E-mail: jwang@htu.cn

2016-04-27 收稿, 2016-06-21 修回, 2016-06-27 接受, 2016-09-21 网络版发表

国家自然科学基金(21403059, 21133009)、河南省基础与前沿技术研究计划(142300413213)和河南省教育厅科学技术研究重点项目 (14A150031)资助

\begin{abstract}
摘要近年来, 化石燃料燃烧产生的二氧化硫 $\left(\mathrm{SO}_{2}\right)$ 对人类健康和生态环境造成严重威胁, 如何有效捕集 $\mathrm{SO}_{2}$ 引 起了国内外学者的广泛关注。离子液体作为一种新型绿色溶剂, 具有蒸汽压低、液程宽、稳定性好、结构和性质 可调节等特殊的性质，在气体分离领域得到了广泛的应用，被视为有前景的 $\mathrm{SO}_{2}$ 吸收剂。调节离子液体的结构，进 一步改善离子液体的捕集性能, 使其快速、高效、低耗、可逆地捕集烟气 $\mathrm{SO}_{2}$ 是研究的关键. 本文综述了近年来离 子液体捕集 $\mathrm{SO}_{2}$ 的研究进展, 主要内容包括常规离子液体和功能离子液体, 其中功能离子液体主要包括有机酸盐 离子液体、含酚基阴离子或唑基阴离子的离子液体、含醚基或氨基的离子液体和多功能离子液体. 同时, 对目前 该领域的发展所面临的主要问题和进一步的研究工作提出了建议.
\end{abstract}

关键词离子液体, 二氧化硫, 硫捕集, 功能化

二氧化硫 $\left(\mathrm{SO}_{2}\right)$ 是大气主要污染物之一, 主要来 源于煤、石油等化石燃料的燃烧. 大气中的 $\mathrm{SO}_{2}$ 经过 空气中的粉尘催化氧化, 形成硫酸型酸雨, 进而对人 类健康和生态环境造成严重危害. 中华人民共和国 环境保护部 “2015年环境状况公报”指出, 尽管与 2010年相比，2015年全国 $\mathrm{SO}_{2}$ 排放总量有所降低, 但 仍然高达 1859.1 万吨 ${ }^{[1]}$. 控制大气中 $\mathrm{SO}_{2}$ 的含量, 主 要是控制 $\mathrm{SO}_{2}$ 的排放. 目前烟气脱硫(flue gas desulfurization, FGD) 被认为是控制 $\mathrm{SO}_{2}$ 排放最有效的方法. 最常用的烟气脱硫方法是石灰石/石灰-石膏法, 虽然 其综合脱硫效率高达 $90 \%$ 以上, 但是生成的低品位 石膏 60\%被废弃, 造成二次污染. 同时, 硫是一种宝 贵的化工资源, 如果将烟气中的 $\mathrm{SO}_{2}$ 进行分离富集, 使其资源化, 意义重大.

离子液体(ionic liquid, IL) 是完全由离子组成且
在 $100^{\circ} \mathrm{C}$ 以下呈液态的有机盐, 本文拟讨论的离子液 体的阳离子和阴离子的结构及缩写见图1. 离子液体 具有很多卓越的性能, 例如: 几乎没有蒸汽压, 不造 成大气污染; 具有很宽的液态温度范围, 热稳定和化 学稳定性好; 对极性和非极性化合物均具有良好的 溶解能力; 导电能力强, 电化学窗口宽; 可设计性 强, 可以根据需要设计出具有特定性能的离子液 体 $^{[2]}$. 由于离子液体的特殊性质, 使其作为溶剂或者 反应催化剂在能源、资源、环境等领域得到广泛关注. 2004 年, 韩布兴课题组 ${ }^{[3]}$ 首次用 $[\mathrm{TMG}][\mathrm{L}]$ 离子 液体对模拟烟气中 $\mathrm{SO}_{2}$ 气体的捕集进行了研究. 他们 发现, 低压下离子液体主要通过化学作用脱除 $\mathrm{SO}_{2}$, 而在高压下既有化学作用亦有物理作用. 随后, 国内 外众多研究人员对离子液体捕集 $\mathrm{SO}_{2}$ 的行为进行了 研究, 新型功能化离子液体不断涌现. 与传统方 
Cation

[Emim]

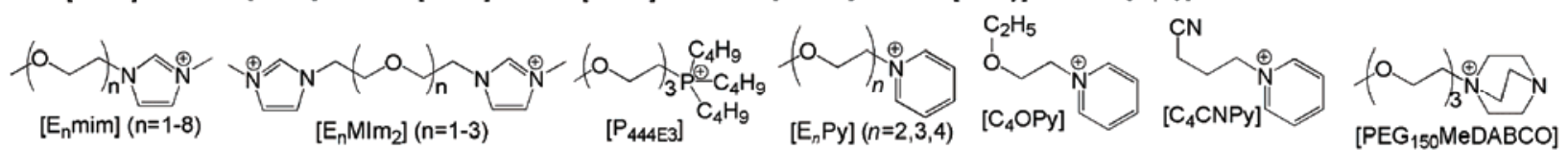

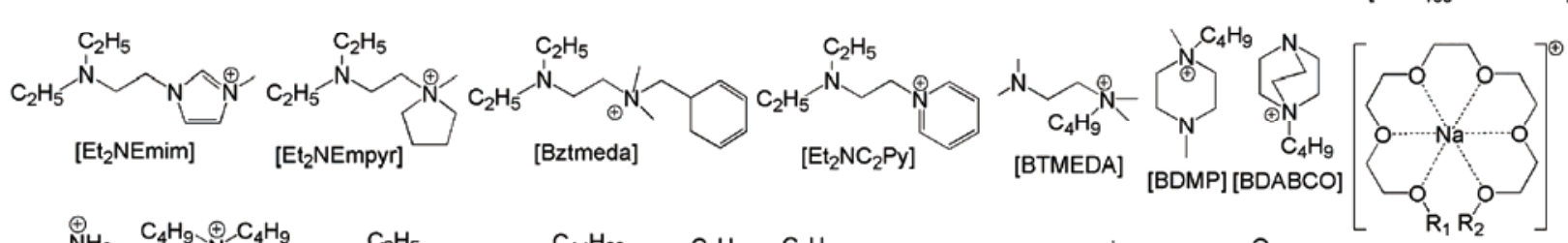

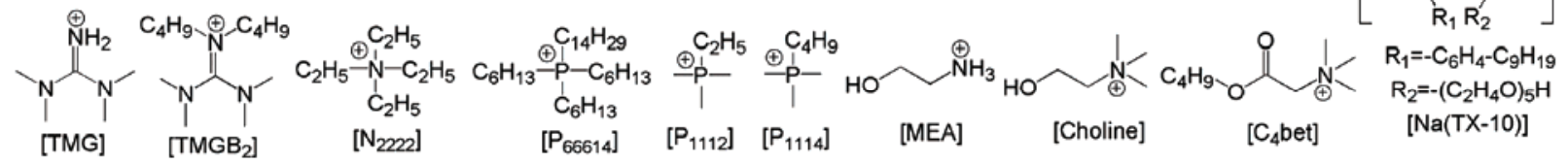

Anion

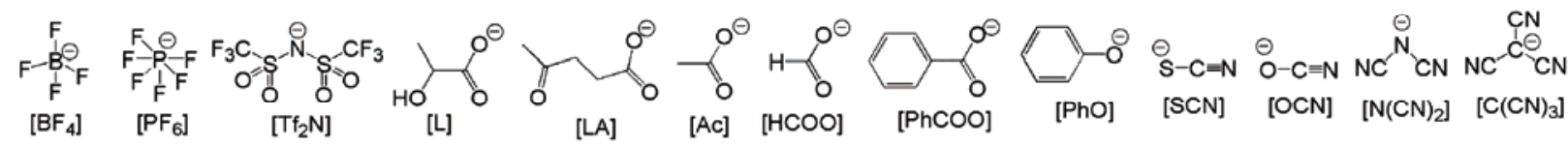

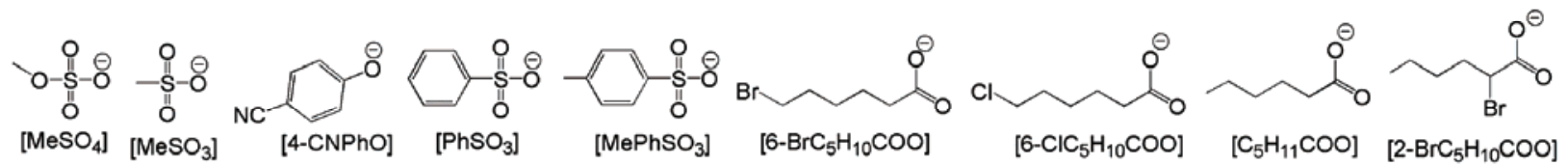

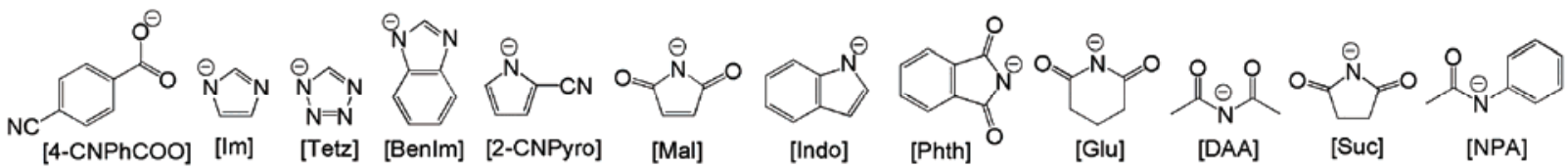

图 1 用于捕集 $\mathrm{SO}_{2}$ 的部分离子液体的阳离子和阴离子的结构

Figure 1 The structures of cations and anions of ILs used in $\mathrm{SO}_{2}$ capture

法相比, 离子液体具有吸收快、捕集效率高、稳定性 好、可以回收利用等优点 ${ }^{[4]}$. 已发表的研究工作表明, 阴离子在离子液体捕集 $\mathrm{SO}_{2}$ 过程中具有关键作用, 阴 离子的共轭酸碱性越强, 离子液体对 $\mathrm{SO}_{2}$ 的捕集效果 越好. 此外, 具有孤对电子的氨基氮原子和醚基氧原 子也促进离子液体对 $\mathrm{SO}_{2}$ 的捕集.

\section{1 常规离子液体}

本文中, 常规离子液体指的是以 $\mathrm{BF}_{4}{ }^{-}, \mathrm{PF}_{6}{ }^{-}$, $\mathrm{Tf}_{2} \mathrm{~N}^{-}, \mathrm{F}^{-}, \mathrm{Cl}^{-}, \mathrm{Br}^{-}, \mathrm{I}^{-}$等为阴离子且阳离子上不含功 能基团的离子液体. 2006年, Brennecke课题组 ${ }^{[5]}$ 测定 了 $25 \sim 60^{\circ} \mathrm{C}, 0 \sim 3.5 \operatorname{bar}(1 \mathrm{bar}=0.1 \mathrm{MPa})$ 下 $\mathrm{SO}_{2}$ 在[Hmim] $\left[\mathrm{Tf}_{2} \mathrm{~N}\right]$ 和 $[\mathrm{HmPy}]\left[\mathrm{Tf}_{2} \mathrm{~N}\right]$ 离子液体中的溶解度, 表明在 $25^{\circ} \mathrm{C}$ 和 $0.1 \mathrm{MPa} 1 \mathrm{~mol}[\mathrm{Hmim}]\left[\mathrm{Tf}_{2} \mathrm{~N}\right]$ 离子液体可以 吸收 $0.916 \mathrm{~mol} \mathrm{SO}_{2}$, 但在 $0.01 \mathrm{MPa}$ 下仅可以吸收 $0.005 \mathrm{~mol}$. 同时, 还测定了 $[\mathrm{Hmim}]\left[\mathrm{Tf}_{2} \mathrm{~N}\right]$ 捕集 $\mathrm{SO}_{2}$ 过 程的偏摩尔焓 $\left(-20.3 \mathrm{~kJ} \mathrm{~mol}^{-1}\right)$. 据此, 他们认为常温 常压下这些离子液体对 $\mathrm{SO}_{2}$ 均为物理捕集. 这是最早 的关于离子液体纯物理吸收 $\mathrm{SO}_{2}$ 的报道.

随后, Riisager课题组 ${ }^{[6]}$ 研究表明, 虽然 [Bmim] $\left[\mathrm{Tf}_{2} \mathrm{~N}\right],[\mathrm{Bmim}]\left[\mathrm{BF}_{4}\right],[\mathrm{TMG}]\left[\mathrm{BF}_{4}\right],\left[\mathrm{TMG}^{\circ}\left[\mathrm{Tf}_{2} \mathrm{~N}\right]\right.$ 和 $\left[\mathrm{TMGB}_{2}\right]\left[\mathrm{Tf}_{2} \mathrm{~N}\right]$ 离子液体在 $20^{\circ} \mathrm{C}$ 和 $0.1 \mathrm{MPa}$ 条件下对 $\mathrm{SO}_{2}$ 的捕集量比较高, 分别为 $1.33,1.50,1.27,1.18$ 和 $1.60 \mathrm{~mol} / \mathrm{mol} \mathrm{IL}$, 但是在 $\mathrm{SO}_{2}$ 分压为 $0.01 \mathrm{MPa}$ 条件下 的捕集量仅分别为 $0.007,0.005,0.064,0.061$ 和 0.080 $\mathrm{mol} / \mathrm{mol} \mathrm{IL}$. 循环实验和核磁共振、红外光谱均表明 
离子液体与 $\mathrm{SO}_{2}$ 之间的作用仅为物理作用.

吴有庭课题组 ${ }^{[7]}$ 报道了 $\mathrm{SO}_{2}$ 在 $[\mathrm{Emim}]\left[\mathrm{BF}_{4}\right]$, $[\mathrm{Bmim}]\left[\mathrm{BF}_{4}\right],[\mathrm{Hmim}]\left[\mathrm{BF}_{4}\right],[\mathrm{Bmim}]\left[\mathrm{PF}_{6}\right],[\mathrm{Bmim}]\left[\mathrm{Tf}_{2} \mathrm{~N}\right]$ 等咪唑类离子液体中的溶解度. 在 $25^{\circ} \mathrm{C}$ 和 $0.1 \mathrm{MPa}$ 条 件下, $1 \mathrm{~mol}$ 上述离子液体可以分别捕集 $1.22,1.33$, $1.41,1.16,1.23 \mathrm{~mol} \mathrm{SO}_{2}$. 研究表明, 这些离子液体捕 集 $\mathrm{SO}_{2}$ 的驱动力属于物理作用, 符合亨利定律, 并可 以计算得到亨利系数. 吴金平课题组 ${ }^{[8]}$ 采用密度泛函 理论理论(DFT) 计算方法研究了 $\mathrm{BF}_{4}^{-}, \mathrm{PF}_{6}^{-}, \mathrm{Tf}_{2} \mathrm{~N}^{-}$对 $\mathrm{SO}_{2}$ 的吸附行为, 证明了这些阴离子与 $\mathrm{SO}_{2}$ 的相互作 用主要是物理作用.

吴卫泽课题组 ${ }^{[9]}$ 将 $\mathrm{SO}_{2}$ 与 $\mathrm{N}_{2}$ 混合得到总压为 0.1 $\mathrm{MPa}$ 的混合模拟气, 分别测定了在 $20^{\circ} \mathrm{C}$ 和不同 $\mathrm{SO}_{2}$ 分 压的条件下, $[\mathrm{Bmim}]\left[\mathrm{BF}_{4}\right]$ 和 $[\mathrm{Bmim}]\left[\mathrm{PF}_{6}\right]$ 对 $\mathrm{SO}_{2}$ 的捕 集量和亨利常数. 结果表明, 当 $\mathrm{SO}_{2}$ 的含量分别为 $100 \%$ 时, $1 \mathrm{~mol}[\mathrm{Bmim}]\left[\mathrm{BF}_{4}\right]$ 和 $[\mathrm{Bmim}]\left[\mathrm{PF}_{6}\right]$ 离子液体 可以分别捕集 1.42 和 $1.17 \mathrm{~mol} \mathrm{SO}_{2}$, 而当 $\mathrm{SO}_{2}$ 的含量降 低至 $0.34 \%$ 时, $1 \mathrm{~mol}[\mathrm{Bmim}]\left[\mathrm{BF}_{4}\right]$ 和 $[\mathrm{Bmim}]\left[\mathrm{PF}_{6}\right]$ 离子 液体只能分别捕集 0.010 和 $0.005 \mathrm{~mol} \mathrm{SO}_{2}$, 说明降低 $\mathrm{SO}_{2}$ 分压, 捕集量会明显下降, 且离子液体对烟气中 $\mathrm{SO}_{2}$ 的吸收量非常小.

Santos课题组 ${ }^{[10]}$ 利用拉曼光谱和分子动力学模 拟对 $[\mathrm{Bmim}][\mathrm{Br}]$ 和 $\mathrm{SO}_{2}$ 之间的相互作用进行了研究. 结果表明, 在与 $\mathrm{SO}_{2}$ 接触后, $[\mathrm{Bmim}][\mathrm{Br}]$ 的物理性质有 了较大的变化, 该变化源于 $\mathrm{Br}^{-} \cdot \mathrm{SO}_{2}$ 之间相互作用产生 的屏蔽效应. 随后, Jung课题组 ${ }^{[11]}$ 系统研究了 [Bmim] $[\mathrm{Cl}],[\mathrm{Bmim}][\mathrm{Br}],[\mathrm{Bmim}][\mathrm{I}],[\mathrm{Emim}][\mathrm{Cl}],[\mathrm{Hmim}][\mathrm{Cl}]$ 和 $[\mathrm{Omim}][\mathrm{Cl}]$ 等咪唑卤盐离子液体对 $\mathrm{SO}_{2}$ 的捕集. 结 果表明, 在 $50{ }^{\circ} \mathrm{C}$ 和 $0.1 \mathrm{MPa}, 1 \mathrm{~mol}$ 这类离子液体可 以捕集1.91 2.19 mol SO . 此外, 当阳离子相同而阴 离子不同时, 离子液体的捕集能力为: $[\mathrm{Bmim}][\mathrm{Br}]>$ $[\mathrm{Bmim}][\mathrm{C} 1]>[\mathrm{Bmim}][\mathrm{I}]$, 且差异较明显. 进一步研究 表明, 该类离子液体对 $\mathrm{SO}_{2}$ 的吸收为物理作用.

Prasad和Senapati ${ }^{[12]}$ 用第一性原理计算的方法在 分子尺度上研究了烟气中 $\mathrm{SO}_{2}, \mathrm{CO}_{2}, \mathrm{~N}_{2}$ 等不同气体与 离子液体的 $\mathrm{BF}_{4}^{-}, \mathrm{PF}_{6}^{-}, \mathrm{NO}_{3}^{-}, \mathrm{F}^{-}, \mathrm{Cl}^{-}, \mathrm{Br}^{-}$等阴离子之 间的相互作用. 发现阴离子与气体分子之间的相互 作用在气体溶解中占主导作用, 尤其是对于极性气 体分子 $\mathrm{SO}_{2}$ 的溶解. 同时, 气体分子的溶解并未对离 子液体的结构造成影响. Mohammadi和Foroutan ${ }^{[13]}$ 利 用分子动力学模拟对 $\mathrm{SO}_{2}$ 气体在 $[\mathrm{Emim}]\left[\mathrm{BF}_{4}\right]$, [Emim] $\left[\mathrm{PF}_{6}\right],[\mathrm{Emim}]\left[\mathrm{NO}_{3}\right],[\mathrm{Emim}][\mathrm{Cl}],[\mathrm{Emim}][\mathrm{Br}]$ 等5种离
子液体中的溶解行为及对离子液体的结构和物理化 学性质进行了研究.

综上所述, 常规离子液体捕集 $\mathrm{SO}_{2}$ 的能力受到 $\mathrm{SO}_{2}$ 分压的影响, 离子液体与 $\mathrm{SO}_{2}$ 之间的物理作用在 捕集过程中占主导位置, 且吸收过程符合亨利定律. 我们将上述常规离子液体进行 $\mathrm{SO}_{2}$ 捕集的主要数据 列在表 $S 1$ 中. 由表 $S 1$ 可见, 虽然常温常压下这些离 子液体对 $\mathrm{SO}_{2}$ 的捕集量较大, 但若用于烟气中低浓度 $\mathrm{SO}_{2}$ 的吸收时则吸收量急剧下降. 其次, 离子液体的 阴离子在捕集 $\mathrm{SO}_{2}$ 过程中占主导地位. 通常, 离子液 体的阴离子 $\mathrm{X}^{-}$所对应的酸 $\mathrm{HX}$ 的酸性越强, 离子液体 对 $\mathrm{SO}_{2}$ 的捕集量越低. 因此设计功能化的离子液体, 提高离子液体对烟气条件下 $\mathrm{SO}_{2}$ 的捕集量具有重要 的意义.

\section{2 功能离子液体}

\section{1 有机酸盐离子液体}

有机酸盐离子液体指的是以羧酸根、磺酸根和硫 氧酸根等为阴离子且阳离子上不含功能基团的离子 液体. 韩布兴课题组 ${ }^{[3]}$ 利用等摩尔中和反应得到 $[\mathrm{TMG}][\mathrm{L}]$, 并首次对该离子液体捕集 $\mathrm{SO}_{2}$ 进行了研 究. 结果表明, 在 $40^{\circ} \mathrm{C}$ 和 $0.12 \mathrm{MPa}, 1 \mathrm{~mol}$ [TMG][L] 可以捕集 $1.7 \mathrm{~mol} \mathrm{SO}_{2}$, 认为该过程包含化学捕集和 物理捕集. 在 $40{ }^{\circ} \mathrm{C}$ 和 $8 \% \mathrm{SO}_{2}$ 的条件下, $1 \mathrm{~mol}$ [TMG] $[\mathrm{L}]$ 仍可以捕集 $0.978 \mathrm{~mol} \mathrm{SO}_{2}$, 他们认为该捕集过程是 化学捕集, 并通过核磁和红外光谱进行了初步验证.

随后, 越来越多的研究人员从不同角度对该离 子液体捕集 $\mathrm{SO}_{2}$ 的行为进行了研究. 李浩然课题 组 ${ }^{[14,15]}$ 采用量化计算和全原子力场两种方法的研究 表明: 阳离子与 $\mathrm{SO}_{2}$ 作用具有较高的Gibbs自由能, 使 得常温常压下反应困难; 阴离子和 $\mathrm{SO}_{2}$ 之间的 $\mathrm{S} \cdots \mathrm{O}$ 作 用力是阳离子和 $\mathrm{SO}_{2}$ 之间的 $\mathrm{N}-\mathrm{H} \cdots \mathrm{O}$ 氢键的 $3.5 \sim 8.8$ 倍. 因此阴离子上带有负电荷的氧原子是该离子液体捕 集 $\mathrm{SO}_{2}$ 过程中的关键因素. Riisager课题组 ${ }^{[6]}$ 的研究证

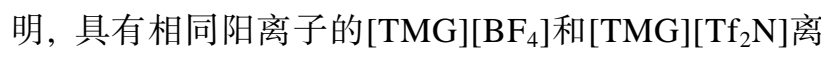
子液体与 $\mathrm{SO}_{2}$ 之间主要是物理作用. $\mathrm{Yu}$ 和 $\mathrm{Chen}^{[16]}$ 采用 从头算对阳离子相同而阴离子不同的 $[\mathrm{TMG}][\mathrm{L}]$,

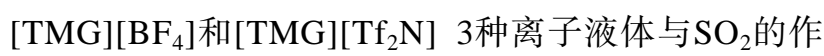
用进行了深人分析, 结果表明, [TMG][L]可以与 $\mathrm{SO}_{2}$

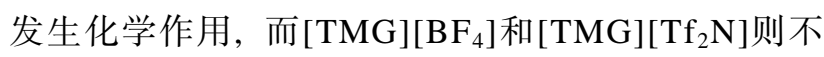
能. 以上研究表明, 阴离子在离子液体与 $\mathrm{SO}_{2}$ 的作用 
中具有重要作用, $[\mathrm{TMG}][\mathrm{L}]$ 是功能离子液体, [TMG] $\left[\mathrm{BF}_{4}\right]$ 和 $[\mathrm{TMG}]\left[\mathrm{Tf}_{2} \mathrm{~N}\right]$ 属于常规离子液体.

张锁江课题组 ${ }^{[17]}$ 以醇胺以及甲酸、乙酸和乳酸 为原料, 合成了一系列醇胺类有机酸盐功能离子液 体, 并研究了这类离子液体对 $\mathrm{SO}_{2}$ 的捕集性能和循环 使用情况. 在 $25^{\circ} \mathrm{C}$ 和0.1 MPa下, $1 \mathrm{~mol}$ [MEA][HCOO], [MEA][Ac]和[MEA][L]离子液体可以分别捕集 0.724 , 0.786 和 $1.041 \mathrm{~mol} \mathrm{SO}_{2}$, 表明该类离子液体对 $\mathrm{SO}_{2}$ 具有 较强的溶解能力. 钟秦课题组 ${ }^{[18]}$ 利用水浴微波法合 成醇胺类离子液体, 并研究了其对 $\mathrm{SO}_{2}$ 的捕集行为. 在 $25^{\circ} \mathrm{C}$ 和 $0.1 \mathrm{MPa}, 1 \mathrm{~mol}$ [MEA][L]离子液体可以吸 收 $1.06 \mathrm{~mol} \mathrm{SO}_{2}$, 且随着循环次数增加, 捕集量越来 越低而脱附剩余量越来越多. 李学良等人 ${ }^{[19]}$ 采用量 子化学计算的方法对醇胺类离子液体与 $\mathrm{SO}_{2}$ 之间的 相互作用进行了研究. 吴有庭课题组以二元羧酸为 阴离子制备了一系列醇胺类离子液体 ${ }^{[20]}$ 和季铵类离 子液体 ${ }^{[21]}$, 并就这些离子液体对 $\mathrm{SO}_{2}$ 的捕集行为进行 了研究.

吴卫泽课题组 ${ }^{[22,23]}$ 研究了阳离子的种类对乳酸 盐离子液体捕集 $\mathrm{SO}_{2}$ 行为的影响. 研究表明, 在 $60^{\circ} \mathrm{C}$ 和0.1 MPa下, $1 \mathrm{~mol}$ [ $\mathrm{N}_{2222}$ [L], [Bmim][L], [Hmim][L], $[\mathrm{TMG}][\mathrm{L}]$ 和 $[\mathrm{MEA}][\mathrm{L}]$ 离子液体可以分别捕集 0.791 , $0.676,0.653,0.414$ 和 $0.230 \mathrm{~mol} \mathrm{SO}_{2}$, 从而说明阳离子 的种类对 $\mathrm{SO}_{2}$ 的捕集行为有很大影响. 通过对 [TMG] [L]和[MEA][L]捕集 $\mathrm{SO}_{2}$ 过程中体系密度、黏度和电导 率变化的研究, 区分了物理作用和化学作用对 $\mathrm{SO}_{2}$ 捕 集的贡献; 对化学反应平衡常数、反应焓变及亨利常 数进行了计算, 提出了离子液体捕集 $\mathrm{SO}_{2}$ 的作用机 理. 此外, 还计算了捕集过程的摩尔Gibbs自由能变、 摩尔焓变以及摩尔熵变. $20^{\circ} \mathrm{C}$ 时, [TMG][L]和[MEA] $[\mathrm{L}]$ 的摩尔反应焓变分别为 -71.78 和 $-47.32 \mathrm{~kJ} \mathrm{~mol}^{-1}$, 说明功能离子液体与 $\mathrm{SO}_{2}$ 之间存在弱化学作用 ${ }^{[9]}$.

随后, 邓东顺课题组 ${ }^{[24]}$ 合成了 2 种以乳酸和乙酰 丙酸为阴离子的可降解胆碱类离子液体. 研究表明, 在 $40^{\circ} \mathrm{C}$ 和 $0.1 \mathrm{MPa}$ 下, $1 \mathrm{~mol}$ [Choline][L]和[Choline] [LA] 可以分别捕集 1.533 和 $1.770 \mathrm{~mol} \mathrm{SO}_{2}$. 当 $\mathrm{SO}_{2}$ 的分 压降低为 $0.0004 \mathrm{MPa}$ 时, $\mathrm{SO}_{2}$ 的溶解量仍然可以达到 0.241 和 $0.274 \mathrm{~mol}$. 测定了离子液体与聚乙二醇 (PEG)复合溶剂对 $\mathrm{SO}_{2}$ 的捕集量, 计算了捕集过程的 摩尔Gibbs自由能变、摩尔焓变以及摩尔熵变. $40^{\circ} \mathrm{C}$ 时, [Choline][L]/ $\mathrm{PEG}_{200}$ 和 [Choline][LA]/ $\mathrm{PEG}_{200}$ 的摩 尔反应焓变分别为 -51.69 和 $-39.81 \mathrm{~kJ} \mathrm{~mol}^{-}$, 说明吸
收是放热过程.

Shiflett和Yokozeki ${ }^{[25]}$ 通过测定 $\mathrm{SO}_{2}$ 在 [Bmim][Ac] 和 $[\mathrm{Bmim}]\left[\mathrm{MeSO}_{4}\right]$ 离子液体中的气液平衡数据, 得到 在 $25^{\circ} \mathrm{C}$ 和 $0.1 \mathrm{MPa}, 1 \mathrm{~mol}$ [Bmim][Ac]和[Bmim] $\left[\mathrm{MeSO}_{4}\right]$ 对 $\mathrm{SO}_{2}$ 的捕集量分别为 1.91 和 $2.11 \mathrm{~mol}$; 当 $\mathrm{SO}_{2}$ 的分压降低为 $0.01 \mathrm{MPa}$ 时, 它们对 $\mathrm{SO}_{2}$ 的溶解量 仍然可以达到 0.664 和 $0.464 \mathrm{~mol}$. 他们认为这些捕集 过程均为化学捕集. Lee等人 ${ }^{[26]}$ 的研究结果表 明, $[\mathrm{Bmim}][\mathrm{Ac}]$ 捕集 $\mathrm{SO}_{2}$ 后再生困难, 而 $[\mathrm{Bmim}]\left[\mathrm{MeSO}_{4}\right]$ 具有中等强度的捕集量且再生性能良好. 同时认为, $\mathrm{SO}_{2}$ 与羧基之间的强相互作用导致含羧基阴离子的 离子液体不能实现可逆捕集. 吕仁庆课题组 ${ }^{[27]}$ 采用 混合密度泛函计算的方法, 对 $[\mathrm{Bmim}]\left[\mathrm{MeSO}_{4}\right]$ 与 $\mathrm{SO}_{2}$, $\mathrm{CO}_{2}$ 之间的相互作用进行了研究. 结果表明, [Bmim] $\left[\mathrm{MeSO}_{4}\right] \cdots \mathrm{SO}_{2}$ 的结构与 $[\mathrm{Bmim}]\left[\mathrm{MeSO}_{4}\right] \cdots \mathrm{CO}_{2}$ 差异很 大，且前者的相互作用能高于后者. 此外, 吴卫泽课 题组 ${ }^{[28]}$ 通过测定含有不同有机酸根的离子液体对 $\mathrm{SO}_{2}$ 的捕集量, 得到如下结论: 离子液体的阴离子对 应有机酸的 $\mathrm{p} K_{\mathrm{a}}$ 值大于亚硫酸的 $\mathrm{p} K_{\mathrm{a}}$ 值时, 该离子液 体可以通过化学作用吸收 $\mathrm{SO}_{2}$.

王从敏课题组 ${ }^{[29]}$ 研究表明, 在 $20^{\circ} \mathrm{C}$ 和 $0.1 \mathrm{MPa}$ 下, $1 \mathrm{~mol}\left[\mathrm{P}_{66614}\right][\mathrm{PhCOO}],\left[\mathrm{P}_{66614}\right][\mathrm{Ac}]$ 和 $\left[\mathrm{P}_{66614}\right]\left[\mathrm{PhSO}_{3}\right]$ 离子液体可以分别捕集 $3.74,3.48$ 和 $3.31 \mathrm{~mol} \mathrm{SO}_{2}$. 同 时对含有脐基的离子液体 $\left[\mathrm{P}_{66614}\right][\mathrm{SCN}],\left[\mathrm{P}_{66614}\right][\mathrm{OCN}]$, [Emim] $[\mathrm{SCN}],[\mathrm{Emim}]\left[\mathrm{C}(\mathrm{CN})_{3}\right]$ 对 $\mathrm{SO}_{2}$ 的捕集性能进 行了研究 ${ }^{[30,31]}$. 结果表明, 这些离子液体均显示了较 高的 $\mathrm{SO}_{2}$ 捕集量以及较好的可再生性. 例如, 在 $20^{\circ} \mathrm{C}$ 和0.1 MPa下, $1 \mathrm{~mol}\left[\mathrm{P}_{66614}\right][\mathrm{SCN}]$ 和 $\left[\mathrm{P}_{66614}\right][\mathrm{OCN}]$ 离 子液体可以分别捕集 3.24 和 $3.64 \mathrm{~mol} \mathrm{SO}_{2}$. 当 $\mathrm{SO}_{2}$ 分压 为 $0.01 \mathrm{MPa}, 1 \mathrm{~mol}\left[\mathrm{P}_{66614}\right][\mathrm{SCN}]$ 和 $\left[\mathrm{P}_{66614}\right][\mathrm{OCN}]$ 离 子液体仍可以分别捕集 1.06 和 $1.50 \mathrm{~mol} \mathrm{SO}$, 表 明 $[\mathrm{SCN}]^{-},[\mathrm{OCN}]^{-}$阴离子与 $\mathrm{SO}_{2}$ 之间为化学作用. 低 浓度等摩尔捕集预示着单位点化学作用. 根据实验 与理论计算的结果, 推断 $[\mathrm{SCN}]^{-}$阴离子上带负电荷 的硫原子与 $\mathrm{SO}_{2}$ 上电正性的硫原子之间存在强烈的 相互作用，如图2所示.

随后, 张锁江课题组 ${ }^{[32]}$ 合成了一系列吡啶类离 子液体, 包括 $\left[\mathrm{C}_{4} \mathrm{Py}\right]\left[\mathrm{BF}_{4}\right],\left[\mathrm{C}_{6} \mathrm{Py}\right]\left[\mathrm{BF}_{4}\right],\left[\mathrm{C}_{8} \mathrm{Py}\right]\left[\mathrm{BF}_{4}\right]$ 和 $\left[\mathrm{C}_{4} \mathrm{Py}\right]\left[\mathrm{Tf}_{2} \mathrm{~N}\right]$ 等常规离子液体和 $\left[\mathrm{C}_{4} \mathrm{Py}\right][\mathrm{SCN}]$ 功能 离子液体, 并用于 $\mathrm{SO}_{2}$ 的吸收. 结果表明, 在 $20^{\circ} \mathrm{C}$ 和 $0.02 \mathrm{MPa}, 1 \mathrm{~mol}\left[\mathrm{C}_{4} \mathrm{Py}\right][\mathrm{SCN}]$ 离子液体可以吸收 $0.73 \mathrm{~mol} \mathrm{SO}_{2}$, 该值比使用常规离子液体 $\left[\mathrm{C}_{4} \mathrm{Py}\right]\left[\mathrm{BF}_{4}\right]$ 


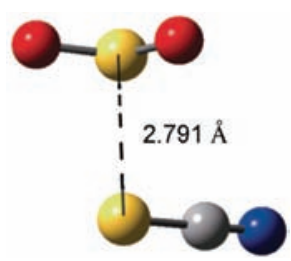

图 2 (网络版彩色) [SCN]功能阴离子与 $\mathrm{SO}_{2}$ 在低浓度下作用的示意 图 $\left(\Delta H=-73.0 \mathrm{~kJ} \mathrm{~mol}^{-1}\right)^{[29]}$

Figure 2 (Color online) The interaction between $[\mathrm{SCN}]$ anion and $\mathrm{SO}_{2}$ under low partial pressure $\left(\Delta H=-73.0 \mathrm{~kJ} \mathrm{~mol}^{-1}\right)^{[29]}$

升高了近0.5 mol. 邓友全课题组 ${ }^{[33]}$ 合成了一系列 以 $[\mathrm{SCN}],\left[\mathrm{N}(\mathrm{CN})_{2}\right],\left[\mathrm{Tf}_{2} \mathrm{~N}\right]$ 为阴离子的甜菜碱类离子 液体，表明在 $20{ }^{\circ} \mathrm{C}$ 和 $\mathrm{SO}_{2}$ 含量为 $10 \%$ 条件下, 1 $\mathrm{mol}\left[\mathrm{C}_{4}\right.$ bet $][\mathrm{SCN}]$ 对 $\mathrm{SO}_{2}$ 的捕集量 $(0.93 \mathrm{~mol})$ 是 $\left[\mathrm{C}_{4}\right.$ bet $]$ $\left[\mathrm{N}(\mathrm{CN})_{2}\right]$ 的2倍, 是常规离子液体 $\left[\mathrm{C}_{4}\right.$ bet $]\left[\mathrm{Tf}_{2} \mathrm{~N}\right]$ 的8倍. 含氰基的有机酸盐离子液体的开发与应用为酸性气 体吸收剂的设计、合成提供了新的思路.

综上所述, 与常规离子液体相比, 有机酸盐离子 液体不仅在常温常压条件下表现出良好的捕集效果, 在较高温度或者低压 $\mathrm{SO}_{2}$ 分压的条件下, 该类离子液 体仍然可以对 $\mathrm{SO}_{2}$ 进行等摩尔捕集, 其最具代表性的 阴离子为 $[\mathrm{L}]$ 和 $[\mathrm{SCN}]$. 根据实验及理论计算结果, 在 较高温度或者较低 $\mathrm{SO}_{2}$ 分压的条件下，该类离子液体 与 $\mathrm{SO}_{2}$ 之间的作用表现出单一位点的化学作用.

\section{2 含酚基阴离子或唑基阴离子的离子液体}

含酚基阴离子的离子液体是指以去除酚羟基上 的氢以后的苯酚类为阴离子的离子液体, 而含唑基 阴离子的离子液体指的是以除去氮原子上的氢之后 的唑类为阴离子的离子液体. 其中苯酚类化合物主 要是指苯酚及各种以酚为命名母体的取代苯酚, 例 如, 卤代苯酚、氧基苯酚等. 唑类化合物主要包括指 咪唑、三氮唑、四唑等共轭氮上含氢的共轭体系. 张 锁江课题组 ${ }^{[34]}$ 以苯酚和 1,1,3,3-四甲基胍为原料合成 了 $[\mathrm{TMG}][\mathrm{PhO}]$ 质子型离子液体, 并研究了其对 $\mathrm{SO}_{2}$ 的捕集行为. 结果表明, 在 $20^{\circ} \mathrm{C}$ 和 $0.1 \mathrm{MPa}, 1 \mathrm{~mol}$ 离子液体可吸收 $2.58 \mathrm{~mol} \mathrm{SO}$. 但是离子液体与 $\mathrm{SO}_{2}$ 的作用力较强, 离子液体再生比较困难. 在 $100^{\circ} \mathrm{C}$ 和 抽真空条件下, 仍有约 $0.75 \mathrm{~mol} \mathrm{SO}_{2}$ 未能脱附. 这大 幅度降低了离子液体的有效捕集量. 王从敏课题 组 ${ }^{[29]}$ 制备了 $\left[\mathrm{P}_{66614}\right][\mathrm{PhO}]$ 非质子型离子液体, 表明在 $20^{\circ} \mathrm{C}$ 和 $0.1 \mathrm{MPa}$ 下, $1 \mathrm{~mol}$ 离子液体可捕集 $3.02 \mathrm{~mol}$ $\mathrm{SO}_{2}$. 进一步研究表明, 与含有苯酚阴离子的离子液
体相比, 含有卤代苯酚阴离子的离子液体不仅可以 提高 $\mathrm{SO}_{2}$ 捕集量, 而且脱附亦容易.

与有机酸盐离子液体和酚基离子液体在低压 $\mathrm{SO}_{2}$ 条件下表现出单一位点的化学作用不同, Wang等 人 $^{[35]}$ 分别以四氮唑和咪唑为阴离子制备了 2 种季鏻 类离子液体 $\left[\mathrm{P}_{66614}\right][\mathrm{Tetz}]$ 和 $\left[\mathrm{P}_{66614}\right][\mathrm{Im}]$. 结果显示, 在 $20^{\circ} \mathrm{C}$ 和 $0.1 \mathrm{MPa}, 1 \mathrm{~mol}$ 离子液体对 $\mathrm{SO}_{2}$ 的捕集量 可以分别达到 3.72 和 $4.80 \mathrm{~mol}$, 且在 $\mathrm{SO}_{2}$ 分压为 0.01 $\mathrm{MPa}$ 条件下的化学捕集量分别为 1.54 和 $2.07 \mathrm{~mol}$. 深 人研究发现, 在 $80{ }^{\circ} \mathrm{C}$ 和 $100 \% \mathrm{~N}_{2}$ 条件下, 吸收 $\mathrm{SO}_{2}$ 后 的 $\left[\mathrm{P}_{66614}\right][\mathrm{Tetz}]$ 容易脱附而 $\left[\mathrm{P}_{66614}\right][\mathrm{Im}]$ 不易脱附, 但 这些离子液体仍具有优良的循环使用性能. 通过理 论计算和谱学手段研究了离子液体和 $\mathrm{SO}_{2}$ 之间的相 互作用，表明这类功能离子液体的唑基阴离子上的 多个电负性较高的 $\mathrm{N}$ 原子与 $\mathrm{SO}_{2}$ 之间存在多位点化学 作用, 如图3所示. 这是低分压条件下实现 $\mathrm{SO}_{2}$ 高效 捕集的主要原因. 因此, 通过增加离子液体与 $\mathrm{SO}_{2}$ 的 作用位点, 来实现高效可逆捕集 $\mathrm{SO}_{2}$ 是非常重要的.

随后，王从敏课题组 ${ }^{[36 ~ 38]}$ 对含唑基阴离子的离 子液体进行了深人研究. 发现含有苯环的唑基离子 液体可以促进对 $\mathrm{SO}_{2}$ 的捕集，其吸收容量可进一步提 高. 在 $20^{\circ} \mathrm{C}$ 和 $0.1 \mathrm{MPa}, 1 \mathrm{~mol}\left[\mathrm{P}_{66614}\right][\mathrm{Im}]$ 可以捕集 $4.80 \mathrm{~mol} \mathrm{SO}_{2}$, 而在阴离子上引入苯环结构后, $1 \mathrm{~mol}$ $\left[\mathrm{P}_{66614}\right][\mathrm{BenIm}]$ 可以捕集 $5.75 \mathrm{~mol} \mathrm{SO}_{2}$; 在 $40^{\circ} \mathrm{C}, \mathrm{SO}_{2}$ 含量为 $500 \mathrm{ppm}\left(1 \mathrm{ppm}=1 \mathrm{~mL} / \mathrm{m}^{3}\right)$ 的烟气条件下, 该离 子液体仍然能捕集约 $1.5 \mathrm{~mol}$ 的 $\mathrm{SO}_{2}$. 进一步考察了阳 离子上引人苯环结构对 $\mathrm{SO}_{2}$ 捕集量的影响, 结果表 明, 在 $20^{\circ} \mathrm{C}$ 和 $0.1 \mathrm{MPa}$ 下, $1 \mathrm{~mol}[\mathrm{Emim}]\left[\mathrm{Tf}_{2} \mathrm{~N}\right]$ 和 [Bzmim] $\left[\mathrm{Tf}_{2} \mathrm{~N}\right]$ 对 $\mathrm{SO}_{2}$ 的捕集量分别为 1.49 和 $1.51 \mathrm{~mol}$, 可见阳离子上苯环结构的引人对离子液体的捕集量 并无明显影响 ${ }^{[36]}$. 理论计算和谱学研究进一步证实, 唑基阴离子上的苯环结构和 $\mathrm{SO}_{2}$ 之间的 $\pi \cdots \mathrm{S}$ 强相互作
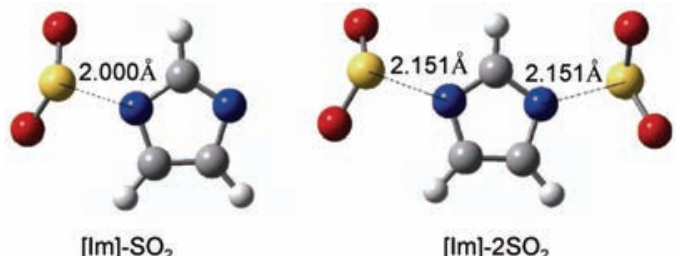

[Im]-2 $\mathrm{SO}_{2}$

图 3 (网络版彩色) [ Im]阴离子与 $\mathrm{SO}_{2}$ 在低分压条件下的多位点相互 作用 $\left(\Delta H_{1}=-124.6 \mathrm{~kJ} \mathrm{~mol}^{-1}, \Delta H_{2}=-75.7 \mathrm{~kJ} \mathrm{~mol}^{-1}\right)^{[34]}$

Figure 3 (Color online) The multiple-site chemical interactions between $[\mathrm{Im}]$ anion and $\mathrm{SO}_{2}$ under low partial pressure $\left(\Delta H_{1}=-124.6 \mathrm{~kJ}\right.$ $\left.\mathrm{mol}^{-1}, \Delta H_{2}=-75.7 \mathrm{~kJ} \mathrm{~mol}^{-1}\right)^{[34]}$ 
用是促进离子液体捕集量增大的重要因素.

王从敏课题组 ${ }^{[39]}$ 还对唑基离子液体捕集 $\mathrm{CO}_{2}$ 进 行了研究, 表明唑基离子液体可以通过调节其碱性, 实现等摩尔捕集 $\mathrm{CO}_{2}$. 鉴于唑基离子液体在捕集 $\mathrm{SO}_{2}$ 过程中表现出的特殊性, 其他研究人员也从不同角 度对该类离子液体捕集 $\mathrm{SO}_{2}$ 的行为进行了进一步研 究. Tang和 $\mathrm{Wu}^{[40]}$ 采用DFT方法对唑基阴离子与 $\mathrm{SO}_{2}$, $\mathrm{CO}_{2}$ 之间的相互作用进行了研究, 表明唑基离子液体 可以通过多位点作用的方式与多个 $\mathrm{SO}_{2}$ 相互作用, 而 至多只能和 1 个 $\mathrm{CO}_{2}$ 相互作用. 在这类唑基离子液体 中, 四唑基阴离子 [Tetz]表现出对于 $\mathrm{SO}_{2}$ 捕集和 $\mathrm{CO}_{2}$ 捕 集不同的性质. 例如, 在 $20^{\circ} \mathrm{C}$ 和 $0.1 \mathrm{MPa}$ 下, $1 \mathrm{~mol}$ [ $\left.\mathrm{P}_{66614}\right]$ [Tetz]仅可以捕集 $0.08 \mathrm{~mol} \mathrm{CO}_{2}{ }^{[35]}$. 邓东顺课题组 ${ }^{[41]}$ 采用DFT方法对 $\left[\mathrm{P}_{1112}\right][\mathrm{Tetz}]$ 和 $\left[\mathrm{P}_{1114}\right][$ Tetz $]$ 离子液体 与 $\mathrm{SO}_{2}, \mathrm{CO}_{2}$ 的作用进行了深人分析. 结果表明, 这些 离子液体的离子对与 $\mathrm{SO}_{2}$ 之间的作用能高于它们与 $\mathrm{CO}_{2}$ 之间的作用能, 且前者电荷之间的转移程度远远 高于后者.

\section{3 含醚基或氨基的离子液体}

含醚基的离子液体指的是阳离子上含有 1 个或者 多个醚氧键 $\left(-\mathrm{CH}_{2} \mathrm{OCH}_{2}-\right.$, 用 $\mathrm{E}_{n}$ 表示, $n$ 为醚基数目)的 离子液体, 该类阳离子易由卤化甘醇单甲醚与甲基 咪唑、吡啶、叔胺等直接合成; 含氨基的离子液体指 的是阳离子或者阴离子上含有伯氨 $\left(-\mathrm{NH}_{2}\right)$ 、仲氨 $\left(-\mathrm{NHR}{ }^{1}\right)$ 、叔氨 $\left(-\mathrm{NR}^{1} \mathrm{R}^{2}\right)$ 等基团的离子液体. 2011年, Jung课题组 ${ }^{[42]}$ 通过将醚氧键引入咪唑阳离子的烷基 链上, 制备了一系列醚基功能化离子液体, 并研究了 醚基对离子液体捕集 $\mathrm{SO}_{2}$ 的影响. 结果表明, 与常规 离子液体相比, 该类离子液体对 $\mathrm{SO}_{2}$ 具有很好的捕集 效果, 并且随着醚氧键个数的增加, $\mathrm{SO}_{2}$ 的溶解量逐步 增大. 在 $30^{\circ} \mathrm{C}$ 和 $0.1 \mathrm{MPa}, 1 \mathrm{~mol}\left[\mathrm{E}_{1} \mathrm{mim}\right]\left[\mathrm{MeSO}_{3}\right.$, $\left[\mathrm{E}_{2} \mathrm{mim}\right]\left[\mathrm{MeSO}_{3}\right],\left[\mathrm{E}_{3} \mathrm{mim}\right]\left[\mathrm{MeSO}_{3}\right]$ 和 $\left[\mathrm{E}_{8} \mathrm{mim}\right]\left[\mathrm{MeSO}_{3}\right]$ 离子液体分别吸收 $2.30,3.18,3.81$ 和 $6.30 \mathrm{~mol} \mathrm{SO}_{2}$, 而 阳离子上不含醚键的乙基甲基咪唑型离子液体 [Emim] $\left[\mathrm{MeSO}_{3}\right]$ 仅可捕集 $1.86 \mathrm{~mol} \mathrm{SO}$. 计算模拟和红外光 谱研究表明, 醚基功能离子液体与 $\mathrm{SO}_{2}$ 之间较强的物 理作用是该类离子液体可以高效捕集 $\mathrm{SO}_{2}$ 的主要 原因.

孙永利课题组 ${ }^{[43]}$ 制备了黏度较低的醚基功能化 双咪唑类离子液体并用于捕集 $\mathrm{SO}_{2}$, 得到了很好的效 果. 研究表明, 在 $40^{\circ} \mathrm{C}$ 和 $0.1 \mathrm{MPa}, 1 \mathrm{~mol}\left[\mathrm{E}_{1} \mathrm{MIm}_{2}\right]$
$\left[\mathrm{Tf}_{2} \mathrm{~N}\right]_{2},\left[\mathrm{E}_{2} \mathrm{MIm}_{2}\right]\left[\mathrm{Tf}_{2} \mathrm{~N}\right]_{2}$ 和 $\left[\mathrm{E}_{3} \mathrm{MIm}_{2}\right]\left[\mathrm{Tf}_{2} \mathrm{~N}\right]_{2}$ 离子液体 可以分别捕集 $1.291,1.506$ 和 $1.620 \mathrm{~mol} \mathrm{SO}_{2}$. 胡永琪课 题组 ${ }^{[44]}$ 研究表明, 在 $25^{\circ} \mathrm{C}$ 和 $0.1 \mathrm{MPa}$ 下, $1 \mathrm{~mol}$ [ $\left.\mathrm{E}_{1} \mathrm{mim}\right]$ $\left[\mathrm{MeSO}_{3}\right],\left[\mathrm{E}_{2} \mathrm{mim}\right]\left[\mathrm{MeSO}_{3}\right]$ 和 $\left[\mathrm{E}_{3} \mathrm{mim}\right]\left[\mathrm{MeSO}_{3}\right]$ 离子液 体附载到 $\mathrm{SiO}_{2}$ 后可以分别捕集 2.621，3.106和3.453 $\mathrm{mol} \mathrm{SO}_{2}$. 此外, 还研究了含有 $\left[\mathrm{MePhSO}_{3}\right]$ 阴离子、不 同链长的醚基阳离子的离子液体对 $\mathrm{SO}_{2}$ 的捕集行 为 ${ }^{[45]}$.

张锁江课题组 ${ }^{[46]}$ 合成了一系列醚基功能化和非 醚基功能化的吡啶类离子液体. 研究表明, 醚基功能 化后, 离子液体易形成室温离子液体. 在 $20^{\circ} \mathrm{C}$ 和 0.1 $\mathrm{MPa}$ 下, $1 \mathrm{~mol}\left[\mathrm{E}_{2} \mathrm{Py}\right][\mathrm{Cl}],\left[\mathrm{E}_{3} \mathrm{Py}\right][\mathrm{Cl}]$ 和 $\left[\mathrm{E}_{4} \mathrm{Py}\right][\mathrm{Cl}]$ 离子 液体可以分别捕集 $3.924,4.289$ 和 $4.594 \mathrm{~mol} \mathrm{SO}_{2}$. 在 相同实验条件下, 与 $\left[\mathrm{E}_{3} \mathrm{Py}\right][\mathrm{Cl}]$ 具有相同数量醚氧键 的 $\left[\mathrm{E}_{3} \mathrm{Mim}\right][\mathrm{Cl}]$ 可以捕集 $4.367 \mathrm{~mol} \mathrm{SO}_{2}$, 且二者捕集 行为高度相似. 魏雄辉课题组 ${ }^{[47]}$ 将一系列甘醇二甲 醚和 $\mathrm{Li}\left[\mathrm{Tf}_{2} \mathrm{~N}\right]$ 混合后得到一类高热稳定性和低挥发性 的金属鳌合型离子液体，并将其应用于 $\mathrm{SO}_{2}$ 捕集，得 到了很好的捕集效果.

需要特别指出的是, 虽然在常温常压条件下, 醚 基离子液体显示出良好的物理捕集效果, 但是在高 温或者低浓度 $\mathrm{SO}_{2}$ 条件下, 这类离子液体捕集 $\mathrm{SO}_{2}$ 的 作用非常有限. Santos课题组 ${ }^{[10]}$ 研究了 [Bmim] [Br] 和 $\mathrm{SO}_{2}$ 之间的相互作用, 认为带有 $-\mathrm{NH}_{2},-\mathrm{NHR}$ 和 $-\mathrm{NR}_{2}$ 等能够作为 $\mathrm{SO}_{2}$ 电子给予体基团的咪唑阳离子能够 提高气体的分离效率. 刘宝友课题组 ${ }^{[48]}$ 制备了 4 种氨 基功能化离子液体, 并将其应用于 $\mathrm{SO}_{2}$ 捕集. 在 $20^{\circ} \mathrm{C}$ 和0.1 MPa下, $1 \mathrm{~mol}$ [ $\left.\mathrm{Et}_{2} \mathrm{NEmim}\right]\left[\mathrm{BF}_{4}\right]$ 和 $\left[\mathrm{Et}_{2} \mathrm{NEmim}\right]$ $\left[\mathrm{PF}_{6}\right]$ 离子液体分别捕集 1.80 和 $1.06 \mathrm{~mol} \mathrm{SO}_{2}$. 阴离子 的结构对吸收效果有较大的影响, 以 $\left[\mathrm{BF}_{4}\right]$ 为阴离子 的离子液体比 $\left[\mathrm{PF}_{6}\right]$ 为阴离子的离子液体具有较高的 吸收效率. 吸收了 $\mathrm{SO}_{2}$ 的离子液体经过简单蒸馏即可 回收使用, 脱附率为 $96 \%$ 。氨基功能化离子液体吸收 $\mathrm{SO}_{2}$ 具有吸收率高、离子液体可以循环使用等优点.

吴卫泽课题组 ${ }^{[49]}$ 合成了 2 种疏水性的氨基功能化 离子液体 $\left[\mathrm{Et}_{2} \mathrm{NEmim}\right]\left[\mathrm{PF}_{6}\right]$ 和 $\left[\mathrm{Et}_{2} \mathrm{NEmpyr}\right]\left[\mathrm{PF}_{6}\right]$, 并应用 于烟气 $\mathrm{SO}_{2}$ 捕集. 当温度为 $30^{\circ} \mathrm{C}$ 时, $1 \mathrm{~mol}\left[\mathrm{Et}_{2} \mathrm{NEmim}\right]$ $\left[\mathrm{PF}_{6}\right]$ 离子液体在 $0.1 \mathrm{MPa}$ 或者 $3 \% \mathrm{SO}_{2}$ 条件下, 可以分 别捕集 2.11 或 $0.94 \mathrm{~mol} \mathrm{SO}$. 氨基功能化离子液体对 $\mathrm{SO}_{2}$ 的高捕集量来源于阳离子上的功能基团 $\left(\mathrm{Et}_{2} \mathrm{NE}\right)$ 与 $\mathrm{SO}_{2}$ 之间的化学作用. 同时, $\left[\mathrm{Et}_{2} \mathrm{NEmim}\right]\left[\mathrm{PF}_{6}\right]$ 是一 种理想的可以循环使用的离子液体. 
Park课题组 ${ }^{[50]}$ 制备了一种氨基功能化的季铵类 离子液体 [Bztmeda] $\left[\mathrm{MeSO}_{3}\right]$, 并通过主成分分析技 术和二维红外相关光谱技术对该离子化合物捕集 $\mathrm{SO}_{2}$ 的行为进行了研究. 结果表明, 在 $30^{\circ} \mathrm{C}$ 和 $0.1 \mathrm{MPa}$ 下, $1 \mathrm{~mol}$ [Bztmeda][ $\mathrm{MeSO}_{3}$ ] 可以捕集 $1.329 \mathrm{~mol} \mathrm{SO}_{2}$. 作者认为氨基上的 2 个氢原子与 $\mathrm{SO}_{2}$ 上的 2 个氧原子 形成的氢键作用是使 [Bztmeda] $\left[\mathrm{MeSO}_{3}\right]$ 可以高效捕 集 $\mathrm{SO}_{2}$ 的主要原因.

Lee课题组 ${ }^{[51]}$ 利用双叔氨基化合物进行单季铵化 反应，合成了 3 种含叔氨基的离子化合物 [BTMEDA] $[\mathrm{Cl}],[\mathrm{BDMP}][\mathrm{Cl}],[\mathrm{BDABCO}][\mathrm{Cl}]$, 并考察了这些盐 的水溶液对 $\mathrm{SO}_{2}$ 的吸收和解吸性能. 谱学和计算结果 表明, 没有被季铵化的叔氨基的碱性对这些盐在水 溶液中捕集 $\mathrm{SO}_{2}$ 具有重要的影响. $\mathrm{SO}_{2}$ 与水反应得到 的 $\mathrm{H}_{2} \mathrm{SO}_{3}$ 再与盐反应, 得到一种以 $\mathrm{HSO}_{3}{ }^{-}$和 $\mathrm{Cl}^{-}$为阴离 子的双阳离子盐溶液.

\section{4 多功能离子液体}

含有单一功能基团的离子液体在捕集 $\mathrm{SO}_{2}$ 过程 中仍显不足, 具体表现在捕集量普遍不高、化学作用 比较强、不易解吸. 在离子液体用于 $\mathrm{SO}_{2}$ 捕集过程中, 以上 2 个问题是研究者普遍关注的问题. 根据离子液 体所具有的可设计性强的优点, 通过调节阴阳离子 的结构, 增加 $\mathrm{SO}_{2}$ 与离子液体之间的作用位点来实现 捕集量的增加, 降低 $\mathrm{SO}_{2}$ 与离子液体之间的强作用焓 来实现易于解吸的目的. 何良年课题组 ${ }^{[52]}$ 以双叔氨 基化合物 $\mathrm{DABCO}$ (三乙烯二胺)为原料, 用三乙二醇单 甲醚进行单季铵化后, 得到碱性离子液体 $\left[\mathrm{E}_{3} \mathrm{DABCO}\right]$ $\left[\mathrm{Tf}_{2} \mathrm{~N}\right]$. 该离子液体具有 2 种功能基团, 分别是醚氧 键以及没有被季铵化的叔氨基. 在 $25^{\circ} \mathrm{C}$ 时, $1 \mathrm{~mol}$ $\left[\mathrm{E}_{3} \mathrm{DABCO}\right]\left[\mathrm{Tf}_{2} \mathrm{~N}\right]$ 离子液体在 $\mathrm{SO}_{2}$ 压力为 $0.1 \mathrm{MPa}$ 或 者 $0.01 \mathrm{MPa}$ 条件下, 可以分别捕集 4.38 或 $1.01 \mathrm{~mol}$ $\mathrm{SO}_{2}$. 常温常压下该离子液体具有高效捕集 $\mathrm{SO}_{2}$ 的 原因, 可能是由于醚氧键具有较强的亲 $\mathrm{SO}_{2}$ 的特性引 起的.

王从敏课题组 ${ }^{[53,54]}$ 开发了一类含有醚氧键的双 功能离子液体, 该类离子液体对 $\mathrm{SO}_{2}$ 具有很强的吸收 能力. 结果表明, 在 $20^{\circ} \mathrm{C}$ 和 $0.1 \mathrm{MPa}, 1 \mathrm{~mol}$ [ $\left.\mathrm{P}_{444 \mathrm{E} 3}\right]$ [Tetz]和 [ $\left.\mathrm{E}_{3} \mathrm{mim}\right][\mathrm{Tetz}]$ 对 $\mathrm{SO}_{2}$ 的可逆捕集容量分别为 5.0 和 $4.43 \mathrm{~mol}$. 当 $\mathrm{SO}_{2}$ 分压下降为 $0.01 \mathrm{MPa}$ 时, 它们 仍可以捕集 1.87 和 $1.58 \mathrm{~mol} \mathrm{SO}_{2}$. 常温常压下醚基与 $\mathrm{SO}_{2}$ 的物理作用效果显著, 而低压下离子液体仅表现
出 $[\mathrm{Tetz}] \cdots \mathrm{SO}_{2}$ 之间的化学作用. 此外, 阳离子为醚基 螯合金属离子的双功能离子液体也可以通过阴阳离 子与 $\mathrm{SO}_{2}$ 的双重作用, 提高 $\mathrm{SO}_{2}$ 捕集量. 例如, 在 $20^{\circ} \mathrm{C}$ 和0.1 MPa下, $1 \mathrm{~mol}$ [Na(TX-10)][Im], [Na(TX-10)] $[\mathrm{PhO}]$ 和 $[\mathrm{Na}(\mathrm{TX}-10)][\mathrm{SCN}]$ 离子液体可以分别捕集 $6.65,5.40$ 和 4.89 mole $\mathrm{SO}_{2}{ }^{[54]}$. 与传统离子液体相比, 这类离子液体合成简单、成本低，并且可以通过改变 金属离子、阴离子、配体大小等来调控离子液体的捕 集、脱附性能.

韩布兴课题组 ${ }^{[55]}$ 合成了一类阳离子含有叔氨 基、阴离子为四唑基的双功能离子液体. 在 $20^{\circ} \mathrm{C}$ 和 0.1 $\mathrm{MPa}$, $1 \mathrm{~mol}\left[\mathrm{Et}_{2} \mathrm{NEmim}\right][\mathrm{Tetz}]$ 和 $\left[\mathrm{Et}_{2} \mathrm{NEmim}_{[}\left[\mathrm{Tf}_{2} \mathrm{~N}\right]\right.$ 对 $\mathrm{SO}_{2}$ 的捕集量分别为 4.32 和 $2.81 \mathrm{~mol}$, 主要原因在 于前者的唑基阴离子与 $\mathrm{SO}_{2}$ 之间存在强烈的相互作 用. 当 $\mathrm{SO}_{2}$ 分压下降为 $0.01 \mathrm{MPa}$ 时, $\left[\mathrm{Et}_{2} \mathrm{NEmim}\right]$ [Tetz] 和 $\left[\mathrm{Et}_{2} \mathrm{NEmim}\right]\left[\mathrm{Tf}_{2} \mathrm{~N}\right]$ 对 $\mathrm{SO}_{2}$ 的捕集量分别为 1.85 和 $0.89 \mathrm{~mol}$, 说明 $\left[\mathrm{Et}_{2} \mathrm{NEmim}\right][\mathrm{Tetz}]$ 的阳离子和阴离子 均能够与 $\mathrm{SO}_{2}$ 进行化学作用. 通过红外及核磁共振波 谱对双功能离子液体 $\left[\mathrm{Et}_{2} \mathrm{NEmim}\right][\mathrm{Tetz}]$ 捕集 $\mathrm{SO}_{2}$ 的机 理进行了研究, 推断 $1 \mathrm{~mol}\left[\mathrm{Et}_{2} \mathrm{NEmim}\right][\mathrm{Tetz}]$ 之所以 能够在低分压条件下捕集约 $2 \mathrm{~mol} \mathrm{SO}_{2}$, 是因为其阳 离子具有含碱性孤对电子的氨基和阴离子具有带负 电荷的氮原子, 这 2 个位点均可以与 $\mathrm{SO}_{2}$ 进行化学作 用, 如图4所示. 随后, 付爱萍课题组 ${ }^{[56]}$ 利用量子化 学计算对 $\left[\mathrm{Et}_{2} \mathrm{NEmim}\right][\mathrm{Tetz}]$ 与 $\mathrm{SO}_{2}$ 之间的相互作用进 行了理论研究.

张锁江课题组 ${ }^{[57]}$ 合成了一系列以 $[\mathrm{SCN}]$ 为阴离子 的吡啶类双功能离子液体 $\left[\mathrm{NEt}_{2} \mathrm{C}_{2} \mathrm{Py}\right][\mathrm{SCN}],\left[\mathrm{C}_{4} \mathrm{CNPy}\right]$ $[\mathrm{SCN}]$ 和 $\left[\mathrm{C}_{4} \mathrm{OPy}\right][\mathrm{SCN}]$. 与单一 $[\mathrm{SCN}]$ 功能化 的 $\left[\mathrm{C}_{4} \mathrm{Py}\right.$. [ $\mathrm{SCN}$ ]离子液体相比, 这些双功能离子液体 对 $\mathrm{SO}_{2}$ 的捕集量较高. 其中, 阳离子含有叔氨基、阴 离子为 $[\mathrm{SCN}]$ 的双功能离子液体 $\left[\mathrm{Et}_{2} \mathrm{NC}_{2} \mathrm{Py}\right][\mathrm{SCN}]$ 对

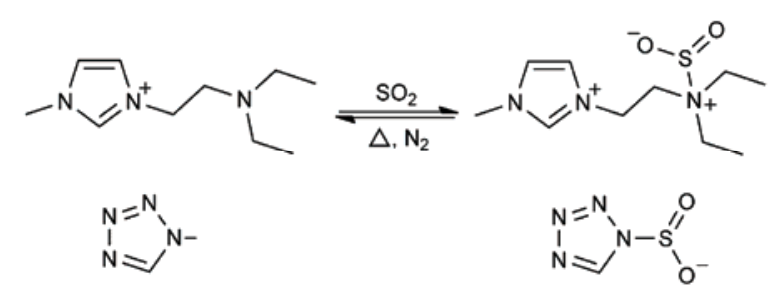

图 $4\left[\mathrm{Et}_{2} \mathrm{NEmim}\right][\mathrm{Tetz}]$ 功能离子液体的结构及与 $\mathrm{SO}_{2}$ 在低分压条件 下相互作用的示意图 ${ }^{[55]}$

Figure 4 The interactions between $\left[\mathrm{Et}_{2} \mathrm{NEmim}\right][\mathrm{Tetz}]$ functionalized $\mathrm{IL}$ and $\mathrm{SO}_{2}$ under low partial pressure ${ }^{[55]}$ 
$\mathrm{SO}_{2}$ 具有良好的捕集效果. 此外, 还就 $\mathrm{SO}_{2}$ 的捕集对 这些离子液体的密度和黏度的影响进行了研究 ${ }^{[58]}$.

针对具有较强化学作用的阴离子不易脱附的问 题, 王从敏课题组 ${ }^{[29,31]}$ 开发了一种双重调节方法来 改善阴离子功能化离子液体对 $\mathrm{SO}_{2}$ 的捕集性能. 他们 在阴离子上引人具有可以与 $\mathrm{SO}_{2}$ 相互作用的卤素、氰 基等吸电子基位点, 来提高离子液体捕集 $\mathrm{SO}_{2}$ 的能力, 同时降低主作用位点的吸收焓, 使脱附容易进行. 例 如, 在 $20^{\circ} \mathrm{C}$ 和0.1 MPa下, $1 \mathrm{~mol}\left[\mathrm{P}_{66614}\right][4-\mathrm{BrPhCOO}]$ 和 $\left[\mathrm{P}_{66614}\right][\mathrm{PhCOO}]$ 对 $100 \% \quad \mathrm{SO}_{2}$ 的捕集量分别为 4.12 和 $3.74 \mathrm{~mol}$. 当脱附条件为 $120^{\circ} \mathrm{C}$ 和 $100 \% \mathrm{~N}_{2}$ 时, 上述 2 种离子液体中 $\mathrm{SO}_{2}$ 的剩余量分别为 0 和 $0.35 \mathrm{~mol}$. DFT 计算表明, [4- $\mathrm{BrPhCOO}] \cdot \mathrm{SO}_{2}$ 之间的作用焓低于 $[\mathrm{PhCOO}] \cdots \mathrm{SO}_{2}$. 除了卤代苯甲酸阴离子外, 卤代苯 酚阴离子、卤代乙酸阴离子和卤代苯酚阴离子等也在 捕集 $\mathrm{SO}_{2}$ 过程中显示出双重作用. 王从敏课题组 ${ }^{[31]}$ 对 一系列氰基功能化的双功能离子液体的阴离子的碱 性与 $\mathrm{SO}_{2}$ 的捕集量、作用焓之间的关系进行了研究. 表 明在 $20^{\circ} \mathrm{C}$ 和0.1 MPa下, $1 \mathrm{~mol}\left[\mathrm{P}_{66614}\right]$ [4-CNPhCOO], [P $\left.{ }_{66614}\right][2-\mathrm{CNPyro}],\left[\mathrm{P}_{66614}\right][\mathrm{OCN}]$ 和 [ $\left.\mathrm{P}_{66614}\right][4-\mathrm{CNPhO}]$ 离子液体可以分别捕集 $3.90,3.74,3.64$ 和 $3.55 \mathrm{~mol}$ $\mathrm{SO}_{2}$, 且在阴离子上引人㲵基后离子液体捕集 $\mathrm{SO}_{2}$ 的 增加量与引人前阴离子的碱性有一定联系, 即原来 的阴离子碱性越强, 其上引人氧基后离子液体捕集 $\mathrm{SO}_{2}$ 的增量越大, 且捕集增量与碱性和吸收焓成线性 关系.

本课题组 ${ }^{[59]}$ 发展了一类酰胺基阴离子双功能化 离子液体, 并研究了其在 $\mathrm{SO}_{2}$ 捕集中的应用. 酰胺基 阴离子就是将酰胺去氢后形成的阴离子, 该阴离子 上除了含有带负电荷的氮原子外还有带电负性的羰基 氧原子. 在 $20^{\circ} \mathrm{C}$ 和 $0.1 \mathrm{MPa}, 1 \mathrm{~mol}\left[\mathrm{P}_{66614}\right]$ [DAA], $\left[\mathrm{P}_{66614}\right][\mathrm{Phth}],\left[\mathrm{P}_{66614}\right][\mathrm{Glu}],\left[\mathrm{P}_{66614}\right][\mathrm{Suc}],\left[\mathrm{P}_{66614}\right][\mathrm{Mal}]$ 和 $\left[\mathrm{P}_{66614}\right][\mathrm{NPA}]$ 离子液体可以分别捕集 4.47, 4.40, $4.12,4.10,4.15$ 和 $3.59 \mathrm{~mol} \mathrm{SO}$. 与 $\left[\mathrm{P}_{66614}\right][\mathrm{Phth}]$ 相比, $20^{\circ} \mathrm{C}$ 和 $0.1 \mathrm{MPa}, 1 \mathrm{~mol}\left[\mathrm{P}_{66614}\right]$ [Indo]的捕集量仅为 $2.86 \mathrm{~mol}$. 当 $\mathrm{SO}_{2}$ 分压为 $0.01 \mathrm{MPa}$ 时, $\left[\mathrm{P}_{66614}\right][\mathrm{Phth}]$ 可 以捕集 $1.87 \mathrm{~mol} \mathrm{SO}_{2}$ 而 $\left[\mathrm{P}_{66614}\right]$ [Indo]仅能捕集 $1.58 \mathrm{~mol}$ $\mathrm{SO}_{2}$. 深人研究表明, $\left[\mathrm{P}_{66614}\right][\mathrm{Phth}]$ 双功能离子液体显 示出的较高捕集量来源于 $\mathrm{C}=\mathrm{O} \cdots \mathrm{S}$ 的相互作用.

此外, 本课题组 ${ }^{[60]}$ 还考察了不同卤素在不同取 代位置时对含有已酸阴离子的离子液体捕集 $\mathrm{SO}_{2}$ 的 影响. 研究表明, 在 $20^{\circ} \mathrm{C}$ 和 $0.1 \mathrm{MPa}, 1 \mathrm{~mol}$ [ $\left.\mathrm{P}_{66614}\right]$
[6- $\left.\mathrm{BrC}_{5} \mathrm{H}_{10} \mathrm{COO}\right],\left[\mathrm{P}_{66614}\right]\left[6-\mathrm{ClC}_{5} \mathrm{H}_{10} \mathrm{COO}\right],\left[\mathrm{P}_{66614}\right][2-\mathrm{B}$ $\mathrm{rC}_{5} \mathrm{H}_{10} \mathrm{COO}$ 和 [ $\left.\mathrm{P}_{66614}\right]\left[\mathrm{C}_{5} \mathrm{H}_{10} \mathrm{COO}\right.$ 离子液体可以分别 捕集 4.34, 4.28, 3.97 和 $3.82 \mathrm{~mol} \mathrm{SO}$. 通过谱学与理论 计算说明, 该类离子液体可以通过卤素与 $\mathrm{COO}^{-}$两种 官能团与 $\mathrm{SO}_{2}$ 作用并获得更高的捕集量, 其中卤素与 $\mathrm{SO}_{2}$ 之间为物理作用, 而 $\mathrm{COO}^{-}$与 $\mathrm{SO}_{2}$ 之间为化学作用. 离子液体经过30次循环捕集, 捕集量没有明显下降.

综上所述, 功能离子液体在常温常压条件下表 现出良好的捕集效果, 具有位点效应, 并且多位点功 能离子液体对 $\mathrm{SO}_{2}$ 的捕集量要高于只具备单一位点 的功能离子液体. 我们将上述功能离子液体进行 $\mathrm{SO}_{2}$ 捕集的主要数据列在表 $\mathrm{S} 2$ 中. 由表 $\mathrm{S} 2$ 可见，通过物 理作用吸收 $\mathrm{SO}_{2}$ 的醚基功能离子液体对低分压 $\mathrm{SO}_{2}$ 的 吸收效果并不好, 但是其他功能离子液体, 不仅在常 温常压下表现出良好的捕集效果，而且可以对低分 压 $\mathrm{SO}_{2}$ 进行有效捕集, 其最具代表性的阴离子 为 [Tetz]和 $[\mathrm{SCN}]$. 当功能离子液体的阴离子 $\mathrm{X}^{-}$所对 应的酸 HX的碱性过强时 (例如 [Im]阴离子), 其所捕 集的 $\mathrm{SO}_{2}$ 因吸收焓较高而难以脱附.

\section{3 总结与展望}

如何根据烟气 $\mathrm{SO}_{2}$ 浓度低且温度高的特点, 设计 开发新型功能离子液体, 实现对烟气中 $\mathrm{SO}_{2}$ 进行快速 高效可逆捕集与利用, 是目前所面临的关键问题. 本 文综述了离子液体捕集 $\mathrm{SO}_{2}$ 气体的研究进展, 重点关 注了离子液体的阴离子在 $\mathrm{SO}_{2}$ 捕集中的关键作用以 及多功能离子液体的研发. 在对上述应用于 $\mathrm{SO}_{2}$ 捕集 的离子液体分析比较后可以得出, 无论是常规离子 液体还是功能离子液体, 阴离子与 $\mathrm{SO}_{2}$ 的作用在离子 液体捕集 $\mathrm{SO}_{2}$ 过程中都是关键的因素. 常规离子液体 一般通过物理作用捕集 $\mathrm{SO}_{2}$, 亨利常数的大小决定了 这类离子液体的捕集性能, 而功能离子液体一般通 过化学作用捕集 $\mathrm{SO}_{2}$, 但作用焓较大时离子液体不易 脱附. 离子液体的碱性与气体吸收焓之间有一定联 系. 最近发展起来的多功能离子液体是根据离子液 体的可设计行强的优点, 将可以与 $\mathrm{SO}_{2}$ 进行化学作用 的官能团和可以与 $\mathrm{SO}_{2}$ 进行物理作用的官能团进行 重组，使离子液体同时具备多个官能团来克服单一 官能团带来的缺点, 既降低了主官能团与 $\mathrm{SO}_{2}$ 之间的 作用焓, 又增加了离子液体的物理捕集量, 最终达到 快速高效可逆低耗捕集的目的.

虽然目前对于离子液体捕集 $\mathrm{SO}_{2}$ 的研究有了较 
大的进展, 我们认为, 要真正将离子液体捕集 $\mathrm{SO}_{2}$ 应 用到工业过程(如火电厂烟气脱硫), 面临的挑战至少 包括以下几个方面：(1) 与传统的石灰石/石灰-石膏 等方法相比, 离子液体尤其是功能化离子液体仍然 具有较高的成本; (2) 对离子液体捕集 $\mathrm{SO}_{2}$ 的机理需 要不断深人和完善, 从而开发出新型 “物美价廉”的 离子液体; (3) 目前离子液体捕集 $\mathrm{SO}_{2}$ 的研究条件一
般都是常温常压, 而烟气的温度比较高, $\mathrm{SO}_{2}$ 的分压 比较低, 因此, 需要进一步研究高温、低分压条件下 离子液体对 $\mathrm{SO}_{2}$ 的捕集; (4) 烟气中除了 $\mathrm{SO}_{2}$ 外, 还有 其他成分, 比如 $\mathrm{CO}_{2}, \mathrm{NO}_{x}, \mathrm{O}_{2}$, 水分和粉尘, 这些组 分对离子液体性能的影响不可忽视. 此外, $\mathrm{CO}_{2}$ 也是 酸性气体, 如何提高离子液体对 $\mathrm{SO}_{2} / \mathrm{CO}_{2}$ 的选择性捕 集具有重要的现实意义.

\section{参考文献}

1 Ministry of Environmental Protection of the people's Republic of China. Report on the State of the Environment in China (in Chinese). http://www.zhb.gov.cn/hjzl/zghjzkgb/lnzghjzkgb [中华人民共和国环境保护部. 2015 中国环境状况公报 (2016-06-01). http://www. zhb.gov.cn/hjzl/zghjzkgb/lnzghjzkgb]

2 Zhang S J, Xu C M, Lü X M. Ionic Liquids and Green Chemistry (in Chinese). Beijing: Science Press, 2009 [张锁江, 徐春明，吕兴梅. 离子液体与绿色化学. 北京: 科学出版社, 2009]

3 Wu W Z, Han B X, Gao H X, et al. Desulfurization of flue gas: $\mathrm{SO}_{2}$ absorption by an ionic liquid. Angew Chem Int Ed, 2004, 43: $2415-2417$

4 Cui G, Wang J, Zhang S. Active chemisorption sites in functionalized ionic liquids for carbon capture. Chem Soc Rev, 2016, doi: $10.1039 / \mathrm{c} 5 \operatorname{cs} 00462 \mathrm{~d}$

5 Anderson J L, Dixon J K, Maginn E J, et al. Measurement of $\mathrm{SO}_{2}$ solubility in ionic liquids. J Phys Chem B, 2006, 110: 15059-15062

6 Huang J, Riisager A, Wasserscheid P, et al. Reversible physical absorption of $\mathrm{SO}_{2}$ by ionic liquids. Chem Commun, 2006, 4027-4029

7 Jiang Y Y, Wu Y T, Zhou Z, et al. $\mathrm{SO}_{2}$ gas separation using supported ionic liquid membranes. J Phys Chem B, 2007, 111: 5058-5061

8 Xing X W, Yao S J, Zhou C G, et al. Density functional theory study on the $\mathrm{SO}_{2}$ adsorption on several ionic liquid anions (in Chinese). J Wuhan Univ (Nat Sci Ed), 2008, 54: 162-166 [邢旭伟，姚淑娟，周成冈，等. 几种常用离子液体阴离子吸附 $\mathrm{SO}_{2}$ 行为的 DFT 计算. 武汉大学学报(理学版), 2008, 54: 162-166]

9 Jin M, Hou Y, Wu W, et al. Solubilities and thermodynamic properties of $\mathrm{SO}_{2}$ in ionic liquids. J Phys Chem B, 2011, 115: 6585-6591

10 Ando R A, Siqueira L J A, Bazito F C, et al. The sulfur dioxide-1-butyl-3-methylimidazolium bromide interaction: Drastic changes in structural and physical properties. J Phys Chem B, 2007, 111: 8717-8719

11 Lee K Y, Kim C S, Kim H, et al. Effects of halide anions to absorb $\mathrm{SO}_{2}$ in ionic liquids. Bull Korean Chem Soc, 2010, 31: 1937-1940

12 Prasad B R, Senapati S. Explaining the differential solubility of flue gas components in ionic liquids from first-principle calculations. J Phys Chem B, 2009, 113: 4739-4743

13 Mohammadi M, Foroutan M. Molecular investigation of $\mathrm{SO}_{2}$ gas absorption by ionic liquids: Effects of anion type. J Mol Liq, 2014, 193: $60-68$

14 Wang Y, Wang C M, Zhang L Q, et al. Difference for $\mathrm{SO}_{2}$ and $\mathrm{CO}_{2}$ in TGML ionic liquids: A theoretical investigation. Phys Chem Chem Phys 2008, 10: 5976-5982

15 Wang Y, Pan H H, Li H R, et al. Force field of the TMGL ionic liquid and the solubility of $\mathrm{SO}_{2}$ and $\mathrm{CO}_{2}$ in the TMGL from molecular dynamics simulation. J Phys Chem B, 2007, 111: 10461-10467

16 Yu G R, Chen X C. SO S $_{2}$ capture by guanidinium-based ionic liquids: A theoretical study. J Phys Chem B, 2011, 115: 3466-3477

17 Yuan X L, Zhang S J, Lu X M. Hydroxyl ammonium ionic liquids: Synthesis, properties, and solubility of $\mathrm{SO}_{2}$. J Chem Eng Data, 2007, 52: 596-599

18 Zhai L Z, Zhong Q, He C, et al. Hydroxyl ammonium ionic liquids synthesized by water-bath microwave: Synthesis and desulfurization. J Hazard Mater, 2010, 177: 807-813

19 Li X L, Chen J J, Luo M, et al. Quantum chemical calculation of hydroxyalkyl ammonium functionalized ionic liquids for absorption $\mathrm{SO}_{2}$ (in Chinese). Acta Phys Chin Sin, 2010, 26: 1364-1372 [李学良, 陈洁洁, 罗梅, 等. 差烷基胺功能化离子液体吸收 $\mathrm{SO}_{2}$ 的量子化学 计算. 物理化学学报, 2010, 26: 1364-1372]

20 Shi L N, Chen M B, Huang K, et al. Performance of MDEA-bicarboxylic acid ionic liquid aqueoussolutions for $\mathrm{SO}_{2}$ capture (in Chinese). CIESC $\mathrm{J}, 2014,65:$ 599-604 [施丽娜, 陈茂兵, 黄宽, 等. MDEA-二元羧酸离子液体溶液吸收/解吸 $\mathrm{SO}_{2}$ 性能. 化工学报, 2014, 65: 599-604]

21 Huang K, Wang G N, Dai Y, et al. Dicarboxylic acid salts as task-specific ionic liquids for reversible absorption of $\mathrm{SO}_{2}$ with a low enthalpy change. RSC Adv, 2013, 3: 16264-16269 
Tian S, Hou Y, Wu W, et al. Absorption of $\mathrm{SO}_{2}$ by thermal-stable functional ionic liquids with lactate anion. RSC Adv, 2013, 3: 3572-3577

Tian S, Hou Y, Wu W, et al. Absorption of $\mathrm{SO}_{2}$ at high temperatures by ionic liquids and the absorption mechanism. Bull Korean Chem Soc, 2014, 35: 2791-2796

Han G Q, Jiang Y T, Deng D S, et al. Absorption of $\mathrm{SO}_{2}$ by renewable ionic liquid/polyethylene glycol binary mixture and thermodynamic analysis. RSC Adv, 2015, 5: 87750-87757

Shiflett M B, Yokozeki A. Chemical absorption of sulfur dioxide in room-temperature ionic liquids. Ind Eng Chem Res, 2010, 49: $1370-1377$

Lee K Y, Gong G T, Song K H, et al. Use of ionic liquids as absorbents to separate $\mathrm{SO}_{2}$ in $\mathrm{SO}_{2} / \mathrm{O}_{2}$ in thermochemical processes to produce hydrogen. Int J Hydrogen Energy, 2008, 33: 6031-6036

$\mathrm{Gu}$ P, Lü R, Wang S, et al. The comparative study on interactions between ionic liquid and $\mathrm{CO}_{2} / \mathrm{SO}_{2}$ by a hybrid density functional approach in the gas phase. Comput Theor Chem, 2013, 1020: 22-31

Ren S, Hou Y, Tian S, et al. What are functional ionic liquids for the absorption of acidic gases? J Phys Chem B, 2013, 117: 2482-2486

Cui G, Zheng J, Luo X, et al. Tuning anion-functionalized ionic iiquids for improved $\mathrm{SO}_{2}$ capture. Angew Chem Int Ed, 2013, 52: $10620-10624$

Wang C, Zheng J, Cui G, et al. Highly efficient $\mathrm{SO}_{2}$ capture through tuning the interaction between anion-functionalized ionic liquids and $\mathrm{SO}_{2}$. Chem Commun, 2013, 49: 1166-1168

Cui G, Zhang F, Zhou X, et al. Tuning the basicity of cyano-containing ionic liquids to improve $\mathrm{SO}_{2}$ capture through cyano-sulfur interaction. Chem Eur J, 2015, 21: 5632-5639

Zeng S, Gao H, Zhang X, et al. Efficient and reversible capture of $\mathrm{SO}_{2}$ by pyridinium-based ionic liquids. Chem Eng J, 2014, 251: 248-256

Yang B, Zhang Q, Fei Y, et al. Biodegradable betaine-based aprotic task-specific ionic liquids and their application in efficient $\mathrm{SO}_{2}$ absorption. Green Chem, 2015, 17: 3798-3805

Shang Y, Li H P, Zhang S J, et al. Guanidinium-based ionic liquids for sulfur dioxide sorption. Chem Eng J, 2011, 175: 324-329

Wang C, Cui G, Luo X, et al. Highly efficient and reversible $\mathrm{SO}_{2}$ capture by tunable azole-based ionic liquids through multiple-site chemical absorption. J Am Chem Soc, 2011, 133: 11916-11919

Cui G, Lin W, Ding F, et al. Highly efficient $\mathrm{SO}_{2}$ capture by phenyl-containing azole-based ionic liquids through multiple-site interactions. Green Chem, 2014, 16: 1211-1216

Cui G. The synthesis of new functionalized ionic liquids and their application in the capture of sulfur dioxide (in Chinese). Doctor Dissertation. Hangzhou: Zhejiang University, 2013 [崔国凯. 新型功能化离子液体的合成及其应用于二氧化硫捕集的研究. 博士学 位论文. 杭州: 浙江大学, 2013]

Chen K, Lin W, Yu X, et al. Designing of anion-functionalized ionic liquids for efficient capture of SO from flue gas. AIChE J, 2015, 61: 2028-2034

Wang C, Luo X, Luo H, et al. Tuning the basicity of ionic liquids for equimolar $\mathrm{CO}_{2}$ capture. Angew Chem Int Ed, 2011, 50: 4918-4922

Tang H, Wu C. Reactivity of azole anions with $\mathrm{CO}_{2}$ from the DFT perspective. ChemSusChem, 2013, 6: 1050-1056

Cui Y H, Chen Y F, Deng D S, et al. Difference for the absorption of $\mathrm{SO}_{2}$ and $\mathrm{CO}_{2}$ on $[$ Pnnnm] [Tetz] $(n=1, m=2$, and 4$)$ ionic liquids: A density functional theory investigation. J Mol Liq, 2014, 199: 7-14

Hong S Y, Im J, Palgunadi J, et al. Ether-functionalized ionic liquids as highly efficient $\mathrm{SO}_{2}$ absorbents. Energy Environ Sci, 2011, 4: 1802-1806

Zhang L, Zhang Z, Sun Y, et al. Ether-functionalized ionic liquids with low viscosity for efficient $\mathrm{SO}_{2}$ capture. Ind Eng Chem Res, 2013, 52: $16335-16340$

Zhao Y, Wang J, Jiang H, et al. Desulfurization performance of ether-functionalized imidazolium-based ionic liquids supported on porous silica gel. Energy Fuels, 2015, 29: 1941-1945

Chen Y, Wang J Y, Liu C, et al. Sulfur dioxide absorption by PEG-based functionalized ionic liquids (in Chinese). CIESC J, 2015, 66 (Suppl): 332-337 [陈莹, 王建英, 刘超, 等. PEG 基功能化离子液体的脱硫性能. 化工学报, 2015, 66(Suppl): 332-337]

Wang J, Zeng S, Bai L, et al. Novel ether-functionalized pyridinium chloride ionic liquids for efficient $\mathrm{SO}_{2}$ capture. Ind Eng Chem Res, 2014, 53: 16832-16839

Sun S, Niu Y, Xu Q, et al. Highly efficient sulfur dioxide capture by glyme-lithium salt ionic liquids. RSC Adv, 2015, 5: 46564-46567

Yang H L, Liu B Y, Wang Y Y. Characterization of amino-functionalized ionic liquids and their application in $\mathrm{SO}_{2}$ absorption (in

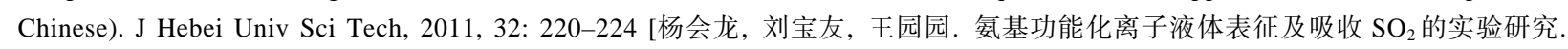
河北科技大学学报, 2011, 32: 220-224]

4 Tian S, Hou Y, Wu W, et al. Hydrophobic task-specific ionic liquids: Synthesis, properties and application for the capture of $\mathrm{SO}_{2}$. $\mathrm{J}$ Hazard Mater, 2014, 278: 409-416 
50 Lee $\mathrm{H}$, Jung $\mathrm{Y} \mathrm{M}$, Lee $\mathrm{K} \mathrm{I}$, et al. Understanding the unique interaction of amine-containing ionic compounds with $\mathrm{SO}_{2}$ for high absorption capacity. RSC Adv, 2013, 3: 25944-25949

51 Lim S R, Hwang J, Kim C S, et al. Absorption and desorption of $\mathrm{SO}_{2}$ in aqueous solutions of diamine-based molten salts. J Hazard Mater, 2015, 289: 63-71

52 Yang Z Z, He L N, Song Q W, et al. Highly efficient $\mathrm{SO}_{2}$ absorption/activation and subsequent utilization by polyethylene glycol-functionalized Lewis basic ionic liquids. Phys Chem Chem Phys, 2012, 14: 15832-15839

53 Cui G, Wang C, Zheng J, et al. Highly efficient $\mathrm{SO}_{2}$ capture by dual functionalized ionic liquids through a combination of chemical and physical absorption. Chem Commun 2012, 48: 2633-2635

54 Ding $\mathrm{F}$, Zheng J, Chen Y, et al. Highly efficient and reversible $\mathrm{SO}_{2}$ capture by surfactant-derived dual functionalized ionic liquids with metal chelate cations. Ind Eng Chem Res, 2014, 53: 18568-18574

55 Yang D, Hou M, Ning H, et al. Reversible capture of $\mathrm{SO}_{2}$ through functionalized ionic liquids. ChemSusChem, 2013, 6: 1191-1195

56 Du D, Fu A, Qin M, et al. $\pi$-Hole interaction: A theoretical insight into the mechanism of $\mathrm{SO}_{2}$ captured by [Et $\mathrm{NEMim}_{2}[\mathrm{Tetz}]$ ionic liquids. J Mol Model, 2015, 21: 1-10

57 Zeng $\mathrm{S}, \mathrm{He} \mathrm{H}$, Gao $\mathrm{H}$, et al. Improving $\mathrm{SO}_{2}$ capture by tuning functional groups on the cation of pyridinium-based ionic liquids. RSC Adv, 2015, 5: 2470-2478

58 Zeng $\mathrm{S}$, Zhang $\mathrm{X}$, Gao $\mathrm{H}$, et al. $\mathrm{SO}_{2}$-induced variations in the viscosity of ionic liquids investigated by in situ fourier transform infrared spectroscopy and simulation calculations. Ind Eng Chem Res, 2015, 54: 10854-10862

59 Cui G, Zhang F, Zhou X, et al. Acylamido-based anion-functionalized ionic liquids for efficient $\mathrm{SO}_{2}$ capture through multiple-site interactions. ACS Sustain Chem Eng, 2015, 3: 2264-2270

60 Cui G, Huang Y, Zhang R, et al. Highly efficient and reversible $\mathrm{SO}_{2}$ capture by halogenated carboxylate ionic liquids. RSC Adv, 2015, 5: 60975-60982

\section{补充材料}

表 $\mathrm{S} 1$ 主要常规离子液体对 $\mathrm{SO}_{2}$ 的摩尔捕集量

表 $\mathbf{S 2}$ 主要功能离子液体对 $\mathrm{SO}_{2}$ 的摩尔捕集量

本文以上补充材料见网络版 csb.scichina.com. 补充材料为作者提供的原始数据, 作者对其学术质量和内容负责. 


\title{
Progress in $\mathrm{SO}_{2}$ capture by ionic liquids
}

\author{
CUI GuoKai, ZHAO Ning, ZHANG FengTao \& WANG JianJi
}

Henan Key Laboratory of Green Chemistry, Collaborative Innovation Center of Henan Province for Green Manu-facturing of Fine Chemicals, Key Laboratory of Green Chemical Media and Reactions, Ministry of Education, School of Chemistry and Chemical Engineering, Henan Normal University, Xinxiang 453007, China

Minimizing the emissions of $\mathrm{SO}_{2}$ is highly important, because it is a significant source of atmospheric pollution that threatens environment and human health. $\mathrm{SO}_{2}$ can be absorbed by ionic liquids (ILs) due to their unique properties, such as ultra low vapor pressure, wide liquid temperature range, non-flammability, chemical stability, and tunable structure and properties. As a new sorption technology, ILs with useful functional groups are attracting the attention of a growing number of scientists and engineers in recent years. In this article, the recent advances on the studies of ILs in the capture of $\mathrm{SO}_{2}$ were reviewed, where traditional ILs and functionalized ILs were included. Traditional ILs with the anions such as $\mathrm{BF}_{4}^{-}, \mathrm{PF}_{6}^{-}, \mathrm{Tf}_{2} \mathrm{~N}^{-}, \mathrm{F}^{-}, \mathrm{Cl}^{-}, \mathrm{Br}^{-}$and $\mathrm{I}^{-}$could uptake $\mathrm{SO}_{2}$ at ambient temperature and pressure through physical interaction between $\mathrm{IL}$ and $\mathrm{SO}_{2}$, but they could not be used under industrial conditions because of the weak interactions. Industrial condition means high temperature and low $\mathrm{SO}_{2}$ partial pressure, which needs strong interaction between $\mathrm{IL}$ and $\mathrm{SO}_{2}$. Functional ILs, including organic acid salt ILs, phenolate ILs, azolate ILs, ether-based ILs, amine-based ILs and multi-functional ILs, showed highly efficient $\mathrm{SO}_{2}$ absorption under ambient conditions. 1,1,3,3-Tetramethylguanidine lactate $([\mathrm{TMG}][\mathrm{L}])$ was the first functional $\mathrm{IL}$ used to achieve about equal mole $\mathrm{SO}_{2}$ capacity under $40^{\circ} \mathrm{C}$ and $8 \% \mathrm{SO}_{2}$ through chemical interaction. [SCN]-based ILs were proved to be highly efficient and reversible ILs for $\mathrm{SO}_{2}$ capture under low partial pressure by the single-site interaction mechanism. Although ether-based ILs could achieve much higher $\mathrm{SO}_{2}$ capacity due to their multiple ether groups, they could not be used to capture $\mathrm{SO}_{2}$ under industrial conditions because of the weak physical interaction. Among the functional ILs investigated, novel azolate ILs were found to have the two mole of chemical absorption capacity through two-site cooperative interactions under low partial pressure. Very recently, other kinds of multiple-site based ILs were developed, including sites on the independent cation and anion as well as those on the same anion. [ $\left.\mathrm{Et}_{2} \mathrm{NEmim}\right][\mathrm{Tetz}]$ was found to be a kind of multi-functional IL that could absorb two mole of $\mathrm{SO}_{2}$ under low partial pressure through independent anion $\cdots \mathrm{SO}_{2}$ and amine $\cdots \mathrm{SO}_{2}$ interactions. Among the above mentioned functional ILs, the ILs based on [Tetz] and [SCN] anions were proved to be more efficient and highly reversible for the capture of $\mathrm{SO}_{2}$ under low $\mathrm{SO}_{2}$ partial pressure. The perspective for further researches on the design of functionalized ILs was also provided. Finally, some challenges in the absorption of $\mathrm{SO}_{2}$ from flue gas were indicated, which includes the high cost of ILs, the deep mechanism study of $\mathrm{SO}_{2}$ absorption, the investigations under high temperature and low $\mathrm{SO}_{2}$ partial pressure, as well as the effect of other species (such as $\mathrm{CO}_{2}, \mathrm{NO}_{x}, \mathrm{O}_{2}$, dust, and moisture) in flue gas. The development of alternative ILs that are able to achieve rapid and reversible $\mathrm{SO}_{2}$ capture in high capacity is always highly desired.

ionic liquids, $\mathrm{SO}_{2}$, sulfur capture, functional

doi: 10.1360/N972016-00534 